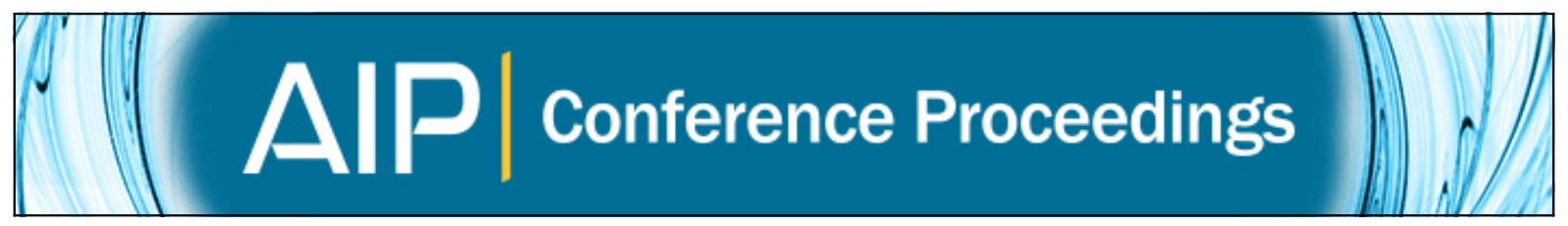

SOHOIACE observations of two consecutive CMEs from the same source region

G. Schettino, S. Dasso, C. H. Mandrini, G. Poletto, and M. Romoli

Citation: AIP Conference Proceedings 1216, 420 (2010); doi: 10.1063/1.3395893

View online: http://dx.doi.org/10.1063/1.3395893

View Table of Contents: http://scitation.aip.org/content/aip/proceeding/aipcp/1216?ver=pdfcov

Published by the AIP Publishing

Articles you may be interested in

Role of latitude of source region in Solar Energetic Particle events

AIP Conf. Proc. 1216, 613 (2010); 10.1063/1.3395941

Solar-Terrestrial Simulations of CMEs with a Realistic Initiation Mechanism: Case Study for Active Region 10069 AIP Conf. Proc. 1216, 440 (2010); 10.1063/1.3395898

STEREO and ACE Observations of Energetic Particles from Corotating Interaction Regions

AIP Conf. Proc. 1216, 379 (2010); 10.1063/1.3395881

Coronal Fine Linear Rays: Are They Fast Streams From Active Regions?

AIP Conf. Proc. 1216, 339 (2010); 10.1063/1.3395869

Quiet solar wind signatures above active regions observed in X-rays

AIP Conf. Proc. 471, 231 (1999); 10.1063/1.58752 


\title{
SOHO/ACE observations of two consecutive CMEs from the same source region
}

\author{
G. Schettino*, S. Dasso ${ }^{\dagger, * *}$, C.H. Mandrini**, G. Poletto ${ }^{\ddagger}$ and M. Romoli* \\ *Dipartimento di Astronomia e Scienza dello Spazio, Università di Firenze, Italy \\ ${ }^{\dagger}$ Departamento de Física, FCEN, Universidad de Buenos Aires, UBA, Buenos Aires, Argentina \\ **Instituto de Astronomía y Física del Espacio, CONICET-UBA, Buenos Aires, Argentina \\ ${ }^{\ddagger} I N A$ F-Osservatorio Astrofisico di Arcetri, Firenze, Italy
}

\begin{abstract}
On June 2, 2003, SOHO/LASCO coronagraph observed two CMEs at the West limb of the Sun, at 00.30 and 08:54 UT, respectively, which appeared to originate from the same source region. Both CMEs show the typical three-part structure. These events have been also observed by SOHO/UVCS, allowing us to infer their physical parameters. We also looked for interplanetary signatures of the CMEs in ACE 'in situ' observations but we did not find evidence of the ejected flux rope; however, the solar wind appeared significantly distorted, probably as a consequence of the influence of both CMEs on their surrounding interplanetary plasma.
\end{abstract}

Keywords: Sun: coronal mass ejections (CMEs) - Sun: UV radiation - Interplanetary Physics: ejecta, driver gasses and magnetic clouds PACS: $96.60 . \mathrm{Ph}$

\section{INTRODUCTION}

Coronal Mass Ejections (CMEs) are sudden expulsions of coronal plasma from the solar surface into the interplanetary space, with speeds that range from hundreds $\mathrm{km} \mathrm{s}^{-1}$ to thousands $\mathrm{km} \mathrm{s}^{-1}$, produced by a loss of equilibrium in the magnetic configuration. Magnetic reconnection is believed to play a fundamental role in the evolution of a CME. As a consequence of the loss of magnetic equilibrium of a flux rope recent models, like the "catastrophe model" (e.g. [15]), predict the formation of a thin current sheet (CS) between the flare loops and the CME bubble. Observational evidence for the presence of a CS has been found (e.g. [5], [12], [20], [16], [2], [6]) both in LASCO [3] and in UVCS [13] observations.

Over the past decade a better knowledge of the physical properties of CMEs and associated CSs has been obtained analyzing spectra acquired by UVCS. Electron temperatures at heliocentric distances of $1.5-1.6 \mathrm{R}_{\odot}$ in the CME front show a wide range of values, from $6 \cdot 10^{3} \mathrm{~K}$ [7] to $2 \cdot 10^{6} \mathrm{~K}$ [2] while values inferred for electron density in the front are of the order of 1.0 . $10^{7} \mathrm{~cm}^{-3}$ at $1.6 \mathrm{R}_{\odot}$ [2]. Evidence of CSs has been detected in UVCS spectra from the emission of high order ions, which are not observed in the quiet corona, like the [Fe XVIII] 974.9 Å line (e.g. [5], [12], [2], [6], [22]). Derived estimates at $1.7 \mathrm{R}_{\odot}$ give typical values for the electron temperature of $(4-6) \cdot 10^{6} \mathrm{~K}$ and for the electron density of $(3-10) \cdot 10^{7} \mathrm{~cm}^{-3}$ [6]. In spite of these results, a lot of questions are still open about the properties of CMEs and associated CSs, like, e.g., the mechanism by which energy is accumulated and what triggers the
CME sudden energy release.

When CMEs are observed 'in situ' in the interplanetary (IP) medium, they are called interplanetary CMEs (ICMEs). These are transient structures that perturb the stationary solar wind as they move away from the Sun. The transit time of an ICME from the Sun to $1 \mathrm{AU}$ is typically in the interval 1 to 5 days [11]. However, if the ICME finds another structure in the solar wind during its travel, it can be slowed down, changing the expected arrival time to Earth because of mutual interaction [8]. ICMEs present several properties significantly different from the typical solar wind; the two most frequently observed are their expansion along the radial direction from the Sun [9] and their low proton temperature [21].

In this paper we study the evolution of two consecutive CMEs that occurred above the same source region. We analyze the physical characteristics of the CME fronts, as observed by UVCS at $1.7 \mathrm{R}_{\odot}$, and we infer the properties of the CS formed after each CME. We also analyze their influence on the solar wind conditions at the Lagrangian point L1 using ACE observations.

\section{LASCO \& UVCS OBSERVATIONS}

The LASCO CME catalog shows that on June 2, 2003, two partial-halo CMEs occurred at 00:30 and 08:54 UT, respectively, above the same source region (active region (AR) NOAA 10365), at position angles (PA), measured counterclockwise from the north solar pole, $261^{\circ}$ and $263^{\circ}$. The measured projected speeds of the two CMEs were $1656 \mathrm{~km} \mathrm{~s}^{-1}$ and $980 \mathrm{~km} \mathrm{~s}^{-1}$, respectively. Movies 


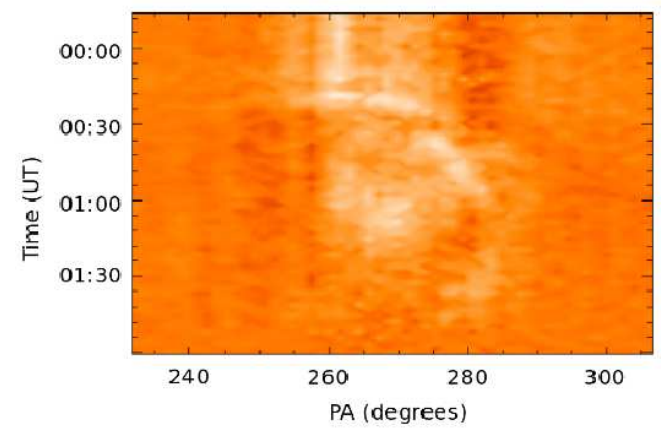

FIGURE 1. Distribution of the O VI $1031.9 \AA$ line intensity vs. PA over a time interval which includes the transit of the CME front through the UVCS slit ( 00:30 UT).

TABLE 1. UVCS configuration during the observations of the two CMEs.

\begin{tabular}{lc}
\hline Heliocentric distance & $1.68 \mathrm{R} \odot$ \\
Position angle & $263^{\circ}$ \\
Slit width & $0.098 \mathrm{~mm}$ \\
Spectral bin size & $0.4 \AA$ \\
Spatial binning & $42^{\prime}$ \\
Observation time & $23: 46$ UT (June 1 2003) - \\
& $02: 05$ UT (June 2); \\
& $06: 08$ UT - 15:58 UT (June 2) \\
\hline
\end{tabular}

of the events can be found in the LASCO website ${ }^{1}$.

On June 2, 2003, also UVCS acquired CME data. The UVCS configuration is shown in Table 1. Both CMEs are detectable in the UVCS spectra from the emission of "cold" ions; the strongest lines are those of the O VI 1031.9-1037.6 $\AA$ doublet, but also the C III $977.0 \AA$ (observed only during the passage of the second CME through the UVCS slit) and the H I Ly $\beta 1025.7 \AA$ (observed in both CMEs) lines are present. Figure 1 shows the distribution of the O VI $1031.9 \AA$ line intensity vs. the position along the UVCS slit, given in terms of PA, over the time interval when the front of the CME crosses the UVCS slit for the 00:30 UT CME; an analogous picture for the 08:54 UT CME is shown in Figure 1 of [22].

\section{ACE OBSERVATIONS}

Solar wind data have been obtained by ACE (at the Lagrangian point L1). The magnetic field observations come from the Magnetic Field Experiment (MAG, [23]) and the plasma data from the Solar Wind Electron Proton Alpha Monitor (SWEPAM, [19]). We explore 'in situ'

\footnotetext{
1 http://cdaw.gsfc.nasa.gov/CME_list/UNIVERSAL/ 2003_06/univ2003_06.html
}

TABLE 2. Physical parameters of the CME fronts.

\begin{tabular}{lcc}
\hline & $\begin{array}{c}\text { CME I } \\
(\mathbf{0 0 : 3 0 ~ U T )})\end{array}$ & $\begin{array}{c}\text { CME II } \\
(\mathbf{0 8 : 5 4} \text { UT })\end{array}$ \\
\hline$T_{e}\left(10^{5} \mathrm{~K}\right)$ & 1.6 & 1.6 \\
$E M\left(10^{25} \mathrm{~cm}^{-5}\right)$ & 0.8 & 5 \\
\hline
\end{tabular}

magnetic and plasma data between June 3 and 7, 2003, to look for evidence of the CMEs on the IP medium.

\section{PHYSICAL PARAMETERS OF THE CMES}

We give here the physical parameters of the fronts and of the CSs associated with the CMEs, as obtained from the analysis of UVCS spectra, and we describe the CME properties, as observed by ACE.

From UVCS data, we derived the electron temperature $T_{e}$ via the line ratio technique:

$$
\frac{I\left(X_{a}\right)}{I\left(Y_{a}\right)}=\frac{A_{X} C\left(T_{e}\right)_{X_{a}}}{A_{Y} C\left(T_{e}\right)_{Y_{a}}}
$$

where $I\left(X_{a}\right)$ and $I\left(Y_{a}\right)$ are the collisional components of the intensities of the ions $X_{a}$ and $Y_{a}, A_{X}$ and $A_{Y}$ are the abundances of the elements $X$ and $Y$, and $C\left(T_{e}\right)$ is the contribution function. Once $T_{e}$ is known, we computed the emission measure $(E M)$ from the equation:

$$
I\left(X_{a}\right)=A_{X} C\left(T_{e}\right)_{X_{a}} E M
$$

where $E M$ is given by the relation $E M=\int N_{e}^{2} d l, N_{e}$ is the electron density and the integration is along the line of sight (LOS). The electron density can then be inferred assuming $E M \simeq N_{e}^{2} L$.

\section{Physical parameters of the CME fronts from UVCS data}

Because of the high speed of the CMEs, we can safely assume that lines in the CME fronts form only by collisional excitation (no radiative component). Hence, we inferred the electron temperature using the ratio $\mathrm{O}$ VI $1031.9 \AA$ to $\mathrm{H}$ I Ly $\beta$ and O VI $1031.9 \AA$ to C III $977.0 \AA$ (only for the second CME), using coronal abundances from [10] and ionization balances from [18]. Once the electron temperature has been determined, we derived the $E M$ for both CMEs. The values obtained are shown in Table 2. 

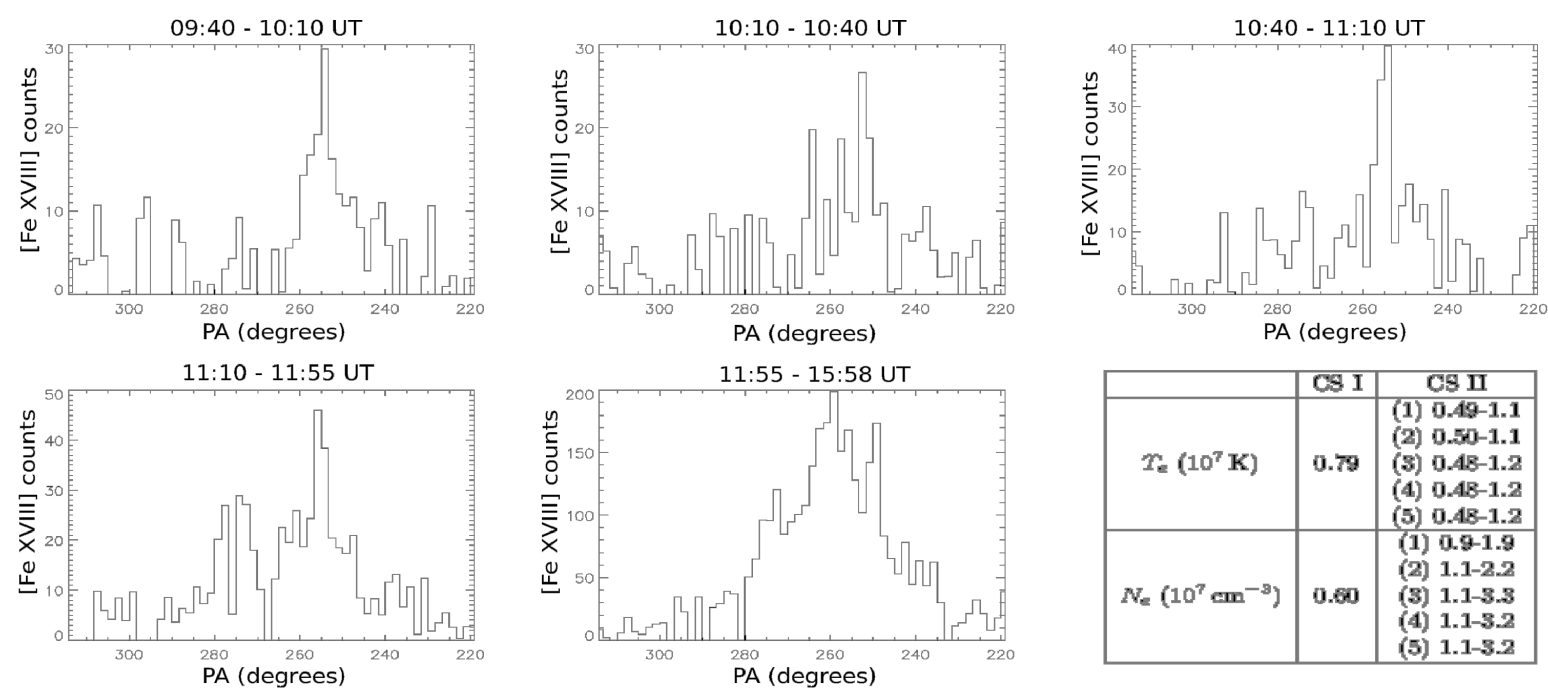

FIGURE 2. [Fe XVIII] $974.9 \AA$ line intensity distribution along the UVCS slit at different times and physical parameters of the CS associated with the two CMEs.

\section{Physical parameters of the CMEs associated CSs from UVCS data}

The CSs that form below both CME bubbles have been detected in the emission from two high order ions, $\mathrm{Si}$ XII $499.4 \AA$ and [Fe XVIII] $974.9 \AA$. Their temperatures and densities have been inferred from the ratio of the intensities of these lines. However, for the first CS our observations started six hours after the CME ejection. At this late time, the CS was very faint and we had to sum over the whole observing interval (two hours) to derive a statistically significant value for temperature and density $\left(T_{e}=7.9 \cdot 10^{6} \mathrm{~K}, N_{e}=6 \cdot 10^{6} \mathrm{~cm}^{-3}\right)$. The second CS was, instead, detectable over a six hour time interval starting about 1 hour after the second CME crossed the UVCS slit; so, we divided UVCS data in five time intervals and studied the CS evolution in time. The distribution of [Fe XVIII] line intensity in the second CS along the UVCS slit is shown in Figure 2: the table gives the inferred temperatures and densities over the five time intervals. Density values have been derived assuming a CS length, along the LOS, $L=8 \cdot 10^{4} \mathrm{~km}$ [24]. We found an ambiguity in the determination of the temperature, and hence of the density, in the second CS due to the concave shape of the $R=\frac{C\left(T_{e}\right)_{S i X I I}}{C\left(T_{e}\right)_{[F e X V I I I]}}$ curve vs. temperature. The minimum of the $R$ curve, $R=1$, corresponds to $T=7.9 \cdot 10^{6} \mathrm{~K}$, while for $1 \leq R \leq 4$ a unique value of the ratio corresponds to two values of the electron temperature. Temperature and density values in Figure 2 are slightly different from those of [22]. Present values represent averages over the whole [Fe XVIII] emission along the UVCS slit, while [22]

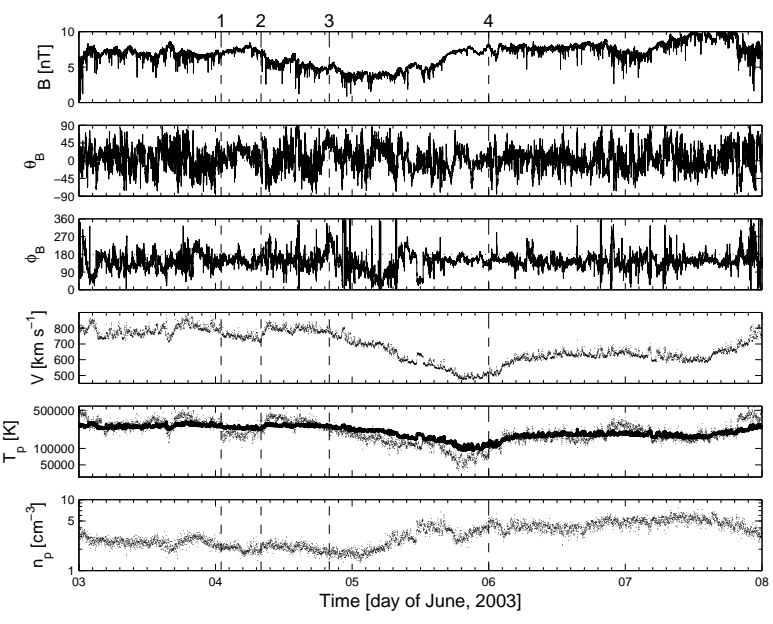

FIGURE 3. In situ (at L1) plasma and magnetic field observations (ACE) for the time window between June 3 and 7, 2003. From top to bottom: absolute value of the magnetic field $(B=|\vec{B}|)$, magnetic field vector orientation in the GSE coordinate system (latitude $\left(\theta_{B}\right)$ and longitude $\left(\phi_{B}\right)$ ), bulk velocity $(V)$, expected (thick continuous line) and observed (dots) proton temperature $\left(T_{p}\right)$, and proton density $\left(n_{p}\right)$. Vertical lines mark different interfaces (see text).

pointed at the fragmented character of the CS and gave separate values of temperature and density in individual CSs appearing at slightly different slit positions. 


\section{The ACE CMEs}

Based on the observed times of the eruptions and their typical expected velocities at $1 \mathrm{AU}$, we explore the interplanetary magnetic field and plasma properties between June 3 and June 7, 2003. Figure 3 shows magnetic and plasma observations for this window.

Two regions (between dashed lines 1-2 and 3-4) can be identified within our time window. In these time intervals, the observed proton temperature $\left(T_{p}\right)$ is clearly lower than the expected temperature for the typical solar wind [21] at a given observed bulk velocity [17]. Another no-Parkerian signature, observed in these intervals, is the almost linear velocity profile along the Sun-Earth direction that indicates a radial expansion from the Sun of the fluid parcels. These non-solar wind features could indicate the presence of two ICMEs. However, ACE data do not reveal any charge state anomalies (see, e.g., [4], [14], [25]) nor regions 1-2, 3-4 appear in the Richardson's ICMEs list, because the observed $T_{p}$ values, although lower than the expected temperature $T_{\text {exp }}$, do not reach the $T_{p} / T_{\exp }$ threshold of 0.5 , hence do not satisfy the empirical criterium that identified ICMEs in Richardson's list. Alternatively these regions correspond to the presence of a solar wind significantly distorted by its interaction with the ICMEs. Taking into account the observed magnetic field (upper panels of Figure 3 ) and the location of the solar source of the transients (west solar limb), we favor the interpretation of regions 1-2 and 34 in terms of the just mentioned interaction. The range 1-2 starts at 01:00 UT of June 4 and its bulk velocity is consistent with an association with the first CME (CME I), while the range 3-4 starts at 20:00 UT of June 4 and its bulk velocity is consistent with an association with the second CME (CME II). We conclude that data provide ambiguous indications and it is difficult to ascribe the non-Parkerian solar wind signatures to a specific process.

\section{DISCUSSION AND CONCLUSIONS}

We have observed remotely two consecutive CMEs originated from the same source region in the Sun and we have analyzed the solar wind parameters observed 'in situ'. We conclude:

(i) The morphology of both CMEs complies with predictions of the "catastrophe model" for the instability and ejection of a coronal flux rope.

(ii) The two CMEs have comparable temperatures $\left(T_{e} \sim\right.$ $\left.2 \cdot 10^{5} \mathrm{~K}\right)$, but their $E M s$ vary by a factor 6 .

(iii) Below both CMEs a CS appears whose temperature $\left(T_{C S}\right)$ ranges between $10^{6.7} \leq T_{C S} \leq 10^{7.2} \mathrm{~K}$, with no significant difference between the two events.
However, the CS of the second CME has a higher $E M$ than the first CME.

(iv) Comparing our $T_{e}$ and $N_{e}$ values with estimates inferred by different authors in other events we conclude that $T_{C S}$ is independent of the event and only densities vary from case to case (see [5], [12], [2], [6]).

(v) The interplanetary manifestation of both CMEs has been identified in ACE data. The plasma velocity and temperature data are somewhat ambiguous and may be interpreted either in terms of ICMEs or of a solar wind significantly perturbed by a transient structure. The timings of both regions identified at $1 \mathrm{AU}$ are consistent with an association with both CMEs, respectively.

\section{ACKNOWLEDGMENTS}

This work was supported by the Argentinean grants: UBACyT X425, UBACyT X127, PICT 2007-1790 and PICT 2007-00856 (ANPCyT). S.D. and C.H.M. are members of the Carrera del Investigador Científico, CONICET. We are greatful to the ACE/SWEPAM and ACE/MAG teams, for the data used for this work.

G. P. acknowledges support from ASI/INAF $\mathrm{I} / 015 / 07 / 0$. SOHO is a project of international collaboration between ESA and NASA.

\section{REFERENCES}

1. A. Bemporad, et al., ApJ, 2006, 638, 1110.

2. A. Bemporad, et al., ApJ, 2007, 655, 576.

3. G. E. Brueckner, et al., Sol. Phys., 1995, 162, 357.

4. H. V. Cane, \& I. G. Richardson, JGR, 2003, 108, 1156.

5. A. Ciaravella, et al., ApJ, 2002, 575, 1116.

6. A. Ciaravella, \& J. C. Raymond, ApJ, 2008, 686, 1372.

7. A. Ciaravella, et al., ApJL, 1997, 491, L59.

8. S. Dasso, et al., JGR, 2009, 114, A02109.

9. P. Démoulin, Solar Phys., 2009, 257, 169.

10. U. Feldman, et al., ApJSS, 1992, 81, 387.

11. N. Gopalswamy, et al., JGR, 2001, 106, 25261.

12. K.-Y. Ko, et al., ApJ, 2003, 594, 1068.

13. J. L. Kohl, et al., Sol. Phys., 1995, 162, 313

14. S. T. Lepri, et al., JGR, 2001, 106, 29231.

15. J. Lin, and T. G. Forbes, JGR, 2000, 105, 2375.

16. J. Lin, et al., ApJ, 2005, 622, 1251.

17. R. E. Lopez, et al., JGR, 1987, 92, 189.

18. P. Mazzotta, et al., A\&ASS, 1998, 133, 403.

19. D. J. McComas, et al., Space Sci. Rev., 1998, 86, 613.

20. J. C. Raymond, et al., ApJ, 2003, 597, 1106.

21. I. J. Richardson and H. V. Cane, JGR, 1995, 100, 23397.

22. G. Schettino, et al., ApJ, 2009, 697, L72.

23. C. W. Smith, et al., Space Sci. Rev., 1998, 86, 613.

24. B. Vršnak, et al., $A \& A, 2009,499,905$.

25. L. Zhao, et al., GRL, 2009, 36, L14104 